\title{
A Comparison of Variable Selection by Tabu Search and Stepwise Regression with Multicollinearity Problem
}

\author{
Kannat Na Bangchang \\ Thammasat University, Pathumthani, Thailand
}

\begin{abstract}
This paper has compared variable selection method for multiple linear regression models that have both relative and non-relative variables in full model when predictor variables are highly correlated 0.999 . In this study two objective functions used in the Tabu Search are mean square error (MSE) and the mean absolute error (MAE). The results of Tabu Search are compared with the results obtained by stepwise regression method based on the hit percentage criterion. The simulations cover the both cases, without and with multicollinearity problems. For each situation, 1,000 iterations are examined by applying a different sample size $n=25$ and 100 at 0.05 level of significance. Without multicollinearity problem, the hit percentages of the stepwise regression method and Tabu Search using the objective function of MSE are almost the same but slightly higher than the Tabu Search using the objective function of MAE. However with multicollinearity problem the hit percentages of the Tabu Search using both objective functions are higher than the hit percentage of the stepwise regression method.
\end{abstract}

Keywords: Stepwise regression, Tabu Search, variable selection

\section{Introduction}

At this time, in many branches of research the use of statistical methods is a necessity if one is to arrive at an overall picture. Inferential statistics are used in the analysis of data in order to summarize the overall features of a population, while at the same time locating the interrelationships among dependent and independent variables. However, there are many methods, depending on the objectives of the research. One popular approach is that of "regression analysis" which is a study of the relationships between independent and dependent variables, in order to explain or predict the values of dependent variables from the independent variables (Montgomery, Peck, \& Vining, 2006).

In the literature, there are many examples of the Tabu Search being used and modified for specific result criteria. The Tabu Search is applied widely such as when Sacchi and Armentano (2011) used compute parametric Tabu Search for solving 0-1 mixed integer programming problems by solving a series of linear programming as weighted terms in the objective function (Sacchi \& Armentano, 2011). Pacheco, Casado, \& Nunez (2009) analyzed for selecting variables that are subsequently used in logistic regression models and found that yields the greatest percentage of hits in logistic regression (Pacheco et al., 2009). Shen, Shi, \& Kong (2010) modified the Tabu Search approach for variable selection in quantitative structure-activity relationship demonstrated that the modified Tabu Search is a tool for variable selection which converges quickly towards

Kannat Na Bangchang, Department of Mathematics and Statistics, Faculty of Science and Technology, Thammasat University, Pathumthani 12121, Thailand. Email: kannat@mathstat.sci.tu.ac.th. 
Regression with Multicollinearity Problem

the optimal position (Shen et al., 2010). Drezner, Marcoulides \& Stohs applied efficiently within finance (Drezner et al., 2001). Finally, Turajlic and Dragovic (2010) used Tabu Search for selecting the set of services with the best overall quality of service by solving the web service selection problem (Turajlic \& Dragovic, 2012). Recently, Wang, Lu \& Glover propose a Tabu Search algorithm for the Unconstrained Binary Quadratic Programming

(Wang et al., 2013). Besides, Mills, Olejnik \& Marcoulides used the Tabu Search procedure for the variable selection (Mills et al., 2005). Consequently, they used Tabu Search in many ways and always used in variable selection.

The validity of applying a linear regression model depends on an appropriate choice of model, requiring a sufficient number of independent variables to explain the dependent variables. But if more independent variables are selected than necessary for application to our model, a large error in prediction will result (Seenoi, 2010). Currently there are many methods for selecting variables, but one method that is extensively used is "stepwise regression". This is a method of choosing independent variables for a regression model. In addition to the technique of selecting variables for use in the method of stepwise regression, there are "combinatorial" techniques for findin $g$ optimal values, which can likewise be used for selecting suitable independent variables, for instance the methods of "Tabu Search" (Glover, 1990) "Simulated Annealing" (Kirkpatrick, 1983) and "Genetic Programming" (Holland, 1975). In this study the Tabu Search is applied to selecting independent variables, comparing the results with that of stepwise regression.

\section{Objectives}

The purpose of this study is to compare the selection of independent variables by the Tabu Search using mean square error (MSE) and the mean absolute error (MAE) with that of stepwise regression, in the multiple linear regression model, by using data that simulates sample sizes of 25 and 100, for data without and with multicollinearity.

\section{Theory}

In this study, the multiple linear regression model is of the form $\mathbf{y}=X \boldsymbol{\beta}+\boldsymbol{\varepsilon}$, where $\mathbf{y}$ is the dependent variable vector, of size $n \times 1, X$ is the matrix of independent variables of size $n \times(k+1), \beta$ is the vector of model parameters, of size $(\mathrm{k}+1) \times 1, \boldsymbol{\varepsilon}$ is the error vector of size $\mathrm{n} \times 1$, subject to the condition $\boldsymbol{\varepsilon} \sim N_{n}\left(0, \sigma^{2} I_{n}\right)$, where $I_{n}$ is an identity matrix of size $\mathrm{n} \times \mathrm{n}, \mathrm{n}$ is the sample size, and $\mathrm{k}$ is the number of independent variables in the model. Since the essential objective of a regression analysis (Bruce, David, \& Richard, 1990) is to arrive at a suitable regression model, which depends on the criteria for selecting variables to be entered in the model, one method that is used extensively is that of stepwise regression.

Stepwise regression is a method for selecting independent variables to be entered into the regression model, by selecting each independent variable that is most closely related to a dependent variable in the regression model. The independent variable entered at each step of the model is checked for its significance. If at any step an independent variable entered into the model is insignificant it is removed. An independent variable entered into the regression model must be tested for its part in explaining variation in the dependent variable. In the case that there are other independent variables in the model (Efroymson, 1960), any independent variable selected for inclusion in the regression model may later be removed if it is found that the variable has no significance. As to specifying the level of significance at which to select an independent 
variable for inclusion in the model $\left(\alpha_{\text {entry }}\right)$, with that at which to remove a variable from the model $\left(\alpha_{\text {stay }}\right)$, these should be specified as of equal value (Smith \& Richard, 1981)

Tabu Search is one method of finding a metaheuristic which is used to find a solution close to the optimum value (Glover, 1990) and which is popularly applied to decision problems, such as the example of the "travelling salesman problem" (Knox, 1989) and "job scheduling". The stages in this procedure are not complicated and the result is highly efficient. Further, a study has been made of methods for finding optimal values of a function for which the variables are continuous, using a Tabu Search for the solution (Siary \& Berthiau, 1997). The Tabu Search for a solution is applied in the selection of the independent variables for a multiple linear regression analysis, finding the highest among the adjusted $\mathrm{R}^{2}\left(R_{a}^{2}\right)$ (Drezner \& George, 1999).

The Tabu Search for a solution can take into consideration more objective functions of varying forms. Though, this method of selecting variables to be entered into the model is different from other methods. Apart from this, studies have been made of the selection of appropriate variables for linear regression analysis by using the methods of searching for on "optimal" value, "mathematical programming", "Lagrangian relaxation" and the "greedy randomized adaptive search procedure" (Eksioglu, Demirer, \& Capar, 2005), while there have been studies of the selection of appropriate models for logistic regression analysis, using the Tabu Search for a solution (Pacheco, Casdo, \& Nunez, 2008).

\section{Method}

This research studies the case of independent variables having a uniform distribution and the error having a normal distribution. There are 6 independent variables in the "full model" and there are 4 independent variables in the model used to find values of the dependent variables, that is the variables $X_{1}, X_{2}, X_{3}$ and $X_{4}$, specifying a population size $\mathrm{N}=100,000$.We set up the independent variables as as $X_{1} \sim U(15,80), X_{2} \sim U(20,150), X_{3} \sim U(10,100), X_{4} \sim U(-50,50), X_{5} \sim U(-100,100)$, and $X_{6} \sim U(-80,400)$. The error is set up to have a normal distribution $\varepsilon \sim N(0,2500)$. We specify the coefficient of regression, which is, $\boldsymbol{\beta}^{\prime}=(100,24,15,-8,5,0,0)$. The sample sizes used are 25 and 100. Apart from this, we specify the correlation coefficient between the independent variables $X_{1}$ and $X_{4}$ equal to 0.999. After that, calculation of the correlation coefficients between $X_{1}$ and $X_{4}$ within each sample are obtained.

Consider the division into groups of randomly obtained samples in accordance with the correlation coefficients between $X_{1}$ and $X_{4}$, as follows:

$0.495 \leq r \leq 0.504$ Classify them into groups with equal correlation coefficients 0.50 .

$0.945 \leq r \leq 0.954$ Classify them into groups with equal correlation coefficients 0.95 .

$0.955 \leq r \leq 0.964$ Classify them into groups with equal correlation coefficients 0.96 .

$0.965 \leq r \leq 0.974$ Classify them into groups with equal correlation coefficients 0.97 .

$0.975 \leq r \leq 0.984$ Classify them into groups with equal correlation coefficients 0.98 .

$0.985 \leq r \leq 0.994$ Classify them into groups with equal correlation coefficients 0.99 .

Repeat this process 1,000 times in each time of correlation coefficient group and sample size.

Later, the selection of a suitable model is undertaken by the method of stepwise regression by specifying the level of significance at which to select an independent variable for entry into the model

$\left(\alpha_{\text {entry }}\right)$ together with that at which to remove a variable from the model $\left(\alpha_{\text {stay }}\right)$, to be equal in value at 0.05 . For a Tabu Search the researchers specified the "Tabu tenure list" as equal to 10, and considered an objective function 


$$
\begin{aligned}
& \text { MSE }= \frac{\sum_{i=1}^{n}\left(y_{i}-\hat{y}_{i}\right)^{2}}{n-k-1} \\
& \text { MAE }=\frac{\sum_{i=1}^{n}\left|y_{i}-\hat{y}_{i}\right|}{n}
\end{aligned}
$$

In consideration of finding the parameter estimators for a regression analysis that provided a minimum value for both objective functions. After that the average mean square error for 1,000 repetitions of the equation for selection of the variables were calculated. Besides, the criteria in this study was to calculate the percentage of the number of times the selection of the correct in variable selection as concluded in Tables 1 and 2.(In Appendix)

\section{Results}

It is found from Table 1, that when the independent variables $\mathrm{X}_{1}$ and $\mathrm{X}_{4}$ have a correlation coefficient of less than 0.1, the method of Tabu Search, using as objective functions the MSE and mean absolute error, correctly selects the independent variables to go into the model at a rate of $88.60 \%$ and $85.60 \%$ respectively. This is similar to the method of stepwise regression, which correctly selects the independent variables to go into the model at a rate of $89.40 \%$

When the independent variables $X_{1}$ and $X_{4}$ have a correlation coefficient in the range 0.495 to 0.504 , the Tabu Search, using as objective functions the MSE and mean absolute error, correctly selects independent variables to go into the model at a rate of $79.40 \%$ and $79.20 \%$ respectively, which is higher than the method of stepwise regression, which correctly selects independent variables to go into the model at a rate of $45.20 \%$.

When the independent variables $X_{1}$ and $X_{4}$ have a correlation coefficient in the range 0.945 to 0.954 , the Tabu Search, using as objective functions the MSE and mean absolute error, correctly selects independent variables to go into the model at a rate of $71.40 \%$ and $70.20 \%$ respectively, which is higher than the method of stepwise regression, which correctly selects independent variables to go into the model at a rate of $15.60 \%$.

When the independent variables $X_{1}$ and $X_{4}$ have a correlation coefficient in the range 0.955 to 0.964 , the Tabu Search, using as objective functions the MSE and mean absolute error, correctly selects independent variables to go into the model at a rate of $70.80 \%$ and $70.00 \%$ respectively, which is higher than the method of stepwise regression, which correctly selects independent variables to go into the model at a rate of $12.40 \%$.

When the independent variables $X_{1}$ and $X_{4}$ have a correlation coefficient in the range 0.965 to 0.974 , the Tabu Search, using as objective functions the MSE and mean absolute error, correctly selects independent variables to go into the model at a rate of $71.00 \%$ and $70.40 \%$ respectively, which is higher than the method of stepwise regression, which correctly selects independent variables to go into the model at a rate of $10.20 \%$.

When the independent variables $X_{1}$ and $X_{4}$ have a correlation coefficient in the range 0.975 to 0.984 , the Tabu Search, using as objective functions the MSE and mean absolute error, correctly selects independent variables to go into the model at a rate of $71.20 \%$ and $69.40 \%$ respectively, which is higher than the method of stepwise regression, which correctly selects independent variables to go into the model at a rate of $9.20 \%$.

When the independent variables $X_{1}$ and $X_{4}$ have a correlation coefficient in the range 0.985 to 0.994 , the 
Tabu Search, using as objective functions the MSE and mean absolute error, correctly selects independent variables to go into the model at a rate of $70.60 \%$ and $69.60 \%$ respectively, which is higher than the method of stepwise regression, which correctly selects independent variables to go into the model at a rate of $7.40 \%$.

Table 1

The mean of regression coefficient and mean square error (MSE) by the correlation coefficient ${ }^{l}$ and the method of variable selection When $\varepsilon_{i} \sim N(0,2500), n=25$

\begin{tabular}{|c|c|c|c|c|c|c|c|c|c|}
\hline & $\mathbf{b}_{0}$ & $\mathbf{b}_{1}$ & $\mathbf{b}_{2}$ & $\mathbf{b}_{3}$ & $\mathbf{b}_{4}$ & $\mathbf{b}_{5}$ & $\mathbf{b}_{6}$ & MSE & $\begin{array}{l}\text { The hit } \\
\text { percentage }\end{array}$ \\
\hline \multicolumn{10}{|l|}{$\mathrm{r}<0.1$} \\
\hline Defined $^{2}$ & 100 & 24 & 15 & -8 & 5 & 0 & 0 & & \\
\hline Tabu_MSE & 99.7028 & 26.2503 & 15.3315 & -10.3452 & 7.5213 & 0.0414 & -0.0223 & 2509.13 & 88.60 \\
\hline Tabu_MAE & 98.9161 & 26.1159 & 15.3436 & -10.2703 & 7.6571 & -0.0817 & -0.0248 & 6002.32 & 85.60 \\
\hline Stepwise & 100.9034 & 23.9646 & 15.0186 & -8.0125 & 5.0032 & 0.0606 & 0.0799 & 2513.25 & 89.40 \\
\hline \multicolumn{10}{|c|}{$0.495 \leq r \leq 0.504$} \\
\hline Defined $^{2}$ & 100 & 24 & 15 & -8 & 5 & 0 & 0 & & \\
\hline Tabu_MSE & 104.3217 & 22.8864 & 15.4021 & -7.0276 & 4.1275 & -0.0898 & 0.0314 & 2511.32 & 79.40 \\
\hline Tabu_MAE & 104.2415 & 22.9238 & 15.4273 & -6.9981 & 4.0321 & 0.0731 & 0.0206 & 6179.11 & 79.20 \\
\hline Stepwise & 59.1386 & 26.7712 & 15.0089 & -8.0095 & 9.4512 & -0.0054 & 0.0201 & 2517.24 & 45.20 \\
\hline \multicolumn{10}{|c|}{$0.945 \leq r \leq 0.954$} \\
\hline Defined $^{2}$ & 100 & 24 & 15 & -8 & 5 & 0 & 0 & & \\
\hline Tabu_MSE & 105.7548 & 20.9375 & 15.4692 & -6.9312 & 3.8820 & -0.1098 & 0.0654 & 2521.52 & 71.40 \\
\hline Tabu_MAE & 105.7425 & 20.9416 & 15.4937 & -6.7558 & 3.6995 & 0.1123 & 0.0456 & 6879.11 & 70.20 \\
\hline Stepwise & -34.1497 & 28.8845 & 15.0109 & -8.0175 & 12.4643 & -0.0181 & 0.0322 & 2523.11 & 15.60 \\
\hline \multicolumn{10}{|c|}{$0.955 \leq r \leq 0.964$} \\
\hline Defined $^{2}$ & 100 & 24 & 15 & -8 & 5 & 0 & 0 & & \\
\hline Tabu_MSE & 104.8070 & 20.9193 & 15.7283 & -6.8772 & 3.6059 & -0.2167 & 0.0568 & 2521.54 & 70.80 \\
\hline Tabu_MAE & 104.7409 & 20.9776 & 15.4020 & -6.7680 & 3.4288 & 0.2389 & 0.0901 & 6880.34 & 70.00 \\
\hline Stepwise & -36.2087 & 29.8401 & 15.0093 & -8.0199 & 15.2276 & -0.1658 & 0.0327 & 2524.17 & 12.40 \\
\hline \multicolumn{10}{|c|}{$0.965 \leq r \leq 0.974$} \\
\hline Defined $^{2}$ & 100 & 24 & 15 & -8 & 5 & 0 & 0 & & \\
\hline Tabu_MSE & 103.7689 & 21.0725 & 15.2612 & -6.7661 & 3.7082 & -0.1176 & 0.0567 & 2521.24 & 71.00 \\
\hline Tabu_MAE & 103.6011 & 21.2781 & 15.3523 & -6.9222 & 3.6174 & 0.0875 & 0.0885 & 6787.11 & 70.40 \\
\hline Stepwise & -48.8832 & 30.6709 & 15.0092 & -8.0156 & 16.9987 & -0.1844 & 0.0717 & 2522.25 & 10.20 \\
\hline \multicolumn{10}{|c|}{$0.975 \leq r \leq 0.984$} \\
\hline Defined $^{2}$ & 100 & 24 & 15 & -8 & 5 & 0 & 0 & & \\
\hline Tabu_MSE & 104.4026 & 21.0057 & 15.7771 & -6.7475 & 3.6870 & -0.0812 & 0.0765 & 2520.52 & 71.20 \\
\hline Tabu_MAE & 107.8179 & 21.4606 & 15.5949 & -7.0149 & 3.7354 & 0.0487 & 0.0875 & 6809.35 & 69.40 \\
\hline Stepwise & -58.6637 & 30.6233 & 15.0132 & -8.0128 & 20.1427 & -0.0774 & 0.0621 & 2524.11 & 9.20 \\
\hline \multicolumn{10}{|c|}{$0.985 \leq r \leq 0.994$} \\
\hline Defined $^{2}$ & 100 & 24 & 15 & -8 & 5 & 0 & 0 & & \\
\hline Tabu_MSE & 104.6102 & 21.2412 & 15.7270 & -6.8583 & 3.8569 & -0.0776 & 0.0342 & 2523.54 & 70.60 \\
\hline Tabu_MAE & 103.8257 & 21.3493 & 15.6940 & -6.5812 & 3.7460 & 0.0648 & 0.0432 & 6802.34 & 69.60 \\
\hline Stepwise & -79.1431 & 31.6635 & 15.0129 & -8.0062 & 20.4422 & -0.0755 & 0.0548 & 2526.12 & 7.40 \\
\hline
\end{tabular}


It is found from Table 2 that, when the independent variables $X_{1}$ and $X_{4}$ have a correlation coefficient of less than 0.1, the method of Tabu Search, using as objective functions the MSE and mean absolute error, correctly selects the independent variables to go into the model at a rate of $90.00 \%$ and $87.20 \%$ respectively, which is similar to the method of stepwise regression, which correctly selects the independent variables to go into the model at a rate of $91.00 \%$

Table 2

The mean of regression coefficient and mean square error (MSE) by the correlation coefficient ${ }^{l}$ and the method of variable selection When $\varepsilon_{i} \sim N(0,2500), n=100$

\begin{tabular}{|c|c|c|c|c|c|c|c|c|c|}
\hline & $\mathbf{b}_{\mathbf{0}}$ & $\mathbf{b}_{1}$ & $\mathbf{b}_{2}$ & $\mathbf{b}_{3}$ & $\mathbf{b}_{4}$ & $\mathbf{b}_{5}$ & $\mathbf{b}_{6}$ & MSE & $\begin{array}{l}\text { The hit } \\
\text { percentage }\end{array}$ \\
\hline \multicolumn{10}{|l|}{$\mathrm{r}<0.1$} \\
\hline Defined $^{2}$ & 100 & 24 & 15 & -8 & 5 & 0 & 0 & & \\
\hline Tabu_MSE & 100.1283 & 25.8183 & 15.1431 & -9.5979 & 7.5622 & 0.0130 & -0.0158 & 2496.23 & 90.00 \\
\hline Tabu_MAE & 99.2788 & 26.0340 & 15.5058 & -10.0237 & 7.9466 & 0.0149 & 0.1054 & 5987.98 & 87.20 \\
\hline Stepwise & 99.7283 & 24.0044 & 15.0022 & -8.0078 & 4.9939 & 0.0377 & 0.0142 & 2499.15 & 91.00 \\
\hline \multicolumn{10}{|c|}{$0.495 \leq r \leq 0.504$} \\
\hline Defined $^{2}$ & 100 & 24 & 15 & -8 & 5 & 0 & 0 & & \\
\hline Tabu_MSE & 105.3216 & 23.0012 & 15.2156 & -7.2897 & 4.4173 & -0.0529 & 0.0219 & 2507.62 & 82.40 \\
\hline Tabu_MAE & 104.9913 & 23.2075 & 15.3071 & -7.1125 & 4.2794 & 0.0698 & 0.0235 & 6109.22 & 81.20 \\
\hline Stepwise & 67.3206 & 26.3129 & 15.0066 & -8.0071 & 9.0315 & -0.0034 & 0.0191 & 2515.13 & 54.80 \\
\hline \multicolumn{10}{|c|}{$0.945 \leq r \leq 0.954$} \\
\hline Defined $^{2}$ & 100 & 24 & 15 & -8 & 5 & 0 & 0 & & \\
\hline Tabu_MSE & 106.2804 & 20.5615 & 15.1781 & -6.7096 & 3.9536 & -0.0678 & 0.0567 & 2497.16 & 74.40 \\
\hline Tabu_MAE & 104.7179 & 21.1603 & 15.2607 & -6.6858 & 3.8422 & 0.0789 & 0.0812 & 6780.35 & 73.20 \\
\hline Stepwise & -14.8497 & 27.3814 & 15.0214 & -8.0178 & 12.4569 & -0.0482 & 0.0315 & 2512.33 & 24.60 \\
\hline \multicolumn{10}{|c|}{$0.955 \leq r \leq 0.964$} \\
\hline Defined $^{2}$ & 100 & 24 & 15 & -8 & 5 & 0 & 0 & & \\
\hline Tabu_MSE & 106.9925 & 20.9708 & 14.7114 & -6.8436 & 3.7251 & -0.0235 & 0.0876 & 2497.01 & 74.60 \\
\hline Tabu_MAE & 104.9351 & 21.0167 & 15.4016 & -6.7386 & 3.7644 & 0.0767 & 0.0689 & 6770.51 & 73.20 \\
\hline Stepwise & -19.2647 & 28.1305 & 15.0221 & -8.0192 & 14.7835 & -0.0859 & 0.0388 & 2502.12 & 22.20 \\
\hline \multicolumn{10}{|c|}{$0.965 \leq r \leq 0.974$} \\
\hline Defined $^{2}$ & 100 & 24 & 15 & -8 & 5 & 0 & 0 & & \\
\hline Tabu_MSE & 107.3514 & 20.9038 & 15.0048 & -6.8593 & 3.7441 & -0.0589 & 0.0812 & 2496.23 & 74.20 \\
\hline Tabu_MAE & 104.0410 & 21.0909 & 15.4366 & -6.9650 & 3.6830 & 0.0598 & 0.0865 & 6712.11 & 73.40 \\
\hline Stepwise & -20.8481 & 30.0913 & 15.0251 & -8.0180 & 15.6083 & -0.0647 & 0.0449 & 2503.17 & 19.80 \\
\hline \multicolumn{10}{|c|}{$0.975 \leq r \leq 0.984$} \\
\hline Defined $^{2}$ & 100 & 24 & 15 & -8 & 5 & 0 & 0 & & \\
\hline Tabu_MSE & 107.5602 & 21.2171 & 15.0951 & -6.7796 & 3.7327 & -0.0768 & 0.0435 & 2495.16 & 75.00 \\
\hline Tabu_MAE & 104.9340 & 20.9719 & 15.5013 & -6.8817 & 3.8298 & 0.0241 & 0.0245 & 6687.35 & 74.60 \\
\hline Stepwise & -28.3969 & 30.5877 & 15.0241 & -8.0197 & 18.8786 & -0.0565 & 0.0313 & 2501.23 & 16.20 \\
\hline \multicolumn{10}{|c|}{$0.985 \leq r \leq 0.994$} \\
\hline Defined $^{2}$ & 100 & 24 & 15 & -8 & 5 & 0 & 0 & & \\
\hline Tabu_MSE & 107.1490 & 21.5488 & 14.9392 & -7.1208 & 3.8325 & -0.0543 & 0.0612 & 2498.34 & 74.80 \\
\hline Tabu_MAE & 105.8325 & 21.3514 & 15.3534 & -7.1485 & 3.7613 & 0.0439 & 0.0786 & 6788.51 & 74.00 \\
\hline Stepwise & -32.8049 & 32.0402 & 15.0209 & -8.0156 & 19.1203 & -0.0371 & 0.0409 & 2503.12 & 12.40 \\
\hline
\end{tabular}

${ }^{1}$ The regression coefficient for simulation

${ }^{2}$ The correlation coefficient between $\mathrm{X}_{1}$ and $\mathrm{X}_{4}$ 
When the independent variables $X_{1}$ and $X_{4}$ have a correlation coefficient in the range 0.495 to 0.504 , the Tabu Search, using as objective functions the MSE and mean absolute error, correctly selects independent variables to go into the model at a rate of $82.40 \%$ and $81.20 \%$ respectively, which is higher than the method of stepwise regression, which correctly selects independent variables to go into the model at a rate of $54.80 \%$.

When the independent variables $X_{1}$ and $X_{4}$ have a correlation coefficient in the range 0.945 to 0.954 , the Tabu Search, using as objective functions the MSE and mean absolute error, correctly selects independent variables to go into the model at a rate of $74.40 \%$ and $73.20 \%$ respectively, which is higher than the method of stepwise regression, which correctly selects independent variables to go into the model at a rate of $24.60 \%$.

When the independent variables $X_{1}$ and $X_{4}$ have a correlation coefficient in the range 0.955 to 0.964 , the Tabu Search, using as objective functions the MSE and mean absolute error, correctly selects independent variables to go into the model at a rate of $74.60 \%$ and $73.20 \%$ respectively, which is higher than the method of stepwise regression, which correctly selects independent variables to go into the model at a rate of $22.20 \%$.

When the independent variables $X_{1}$ and $X_{4}$ have a correlation coefficient in the range 0.965 to 0.974 , the Tabu Search, using as objective functions the MSE and mean absolute error, correctly selects independent variables to go into the model at a rate of $74.20 \%$ and $73.40 \%$ respectively, which is higher than the method of stepwise regression, which correctly selects independent variables to go into the model at a rate of $19.80 \%$.

When the independent variables $X_{1}$ and $X_{4}$ have a correlation coefficient in the range 0.975 to 0.984 , the Tabu Search, using as objective functions the MSE and mean absolute error, correctly selects independent variables to go into the model at a rate of $75.00 \%$ and $74.60 \%$ respectively, which is higher than the method of stepwise regression, which correctly selects independent variables to go into the model at a rate of $16.20 \%$.

When the independent variables $X_{1}$ and $X_{4}$ have a correlation coefficient in the range 0.985 to 0.994 , the Tabu Search, using as objective functions the MSE and mean absolute error, correctly selects independent variables to go into the model at a rate of $74.80 \%$ and $74.00 \%$ respectively, which is higher than the method of stepwise regression, which correctly selects independent variables to go into the model at a rate of $12.40 \%$.

\section{Discussion}

When comparing the efficiency of selection of models, as the sample size increases, in the selection of independent variables by the method of Tabu Search using either of the two objective functions and by the method of stepwise regression, the percentage of models correctly selected increases., This occurs in cases where the data have or do not have multicollinearity.

When comparing the efficiency of selection of independent variables as to whether the data have multicollinearity, it is found that when the correlation coefficient among the independent variables $\mathrm{X}_{1}$ and $\mathrm{X}_{4}$ increases, the method of selection by Tabu Search, with either of the two objective functions, the percentage of models correctly selected is similar. In the case of stepwise regression the percentage of models correctly selected is lower.

With the estimation of values by the method of least squares (OLS), when the independent variables have a multicollinearity problem, it is found that the estimated value for the regression coefficient for the related independent variables trends away from the value specified in the model and is higher than the value of the correlation coefficient for the independent variables obtained by Tabu Search, whether the MSE or the mean absolute error (MAE) is used as the objective function. This is because Tabu Search method does not use $\left(X^{\prime} X\right)^{-1}$ for an estimate parameter leading to error in estimation. (Piriyakul, 1986) However, there is one caveat 
to this method. The standard error in the estimated value, in the case that the independent variables show a multicollinearity problem, is higher than in the case where they do not, and the value of the standard error as obtained from the Tabu Search is of a similar character to that from the method of stepwise regression, in consequence of using the OLS formula for calculation.

However the MSE value obtained with either of the two objective functions causes the calculated value to be lower than it should be. Thus it will make it possible to report that, in the case where the data have a multicollinearity problem, the Tabu Search will enable the researcher to estimate values for the regression coefficient closer to the model parameters than does the OLS method. Apart from this, it can be better used in the estimation of the regression coefficient than can the method of "ridge egression," since it is not necessary to use judgment when deciding on the "ridge trace." Moreover, this is like a robust form of redundancy analysis, seeking directions in the factor space that are associated with high variation in the responses but biasing them toward directions that are accurately predicted. (Gujarati, 2009)

\section{Conclusion}

We define terms as follows:

For the independent variables $\mathrm{X}_{1}$ and $\mathrm{X}_{4}$ to have a low level multicollinearity means that the correlation coefficient among independent variables $\mathrm{X}_{1}$ and $\mathrm{X}_{4}$ has a value less than 0.1 .

For the independent variables $\mathrm{X}_{1}$ and $\mathrm{X}_{4}$ to have a medium level multicollinearity means that the correlation coefficient among independent variables $\mathrm{X}_{1}$ and $\mathrm{X}_{4}$ has a value of 0.5 .

For the independent variables $\mathrm{X}_{1}$ and $\mathrm{X}_{4}$ to have a high level multicollinearity means that the correlation coefficient among independent variables $\mathrm{X}_{1}$ and $\mathrm{X}_{4}$ has a value of $0.95,0.96,0.97,0.98$ or 0.99 .

In the case that the independent variables $\mathrm{X}_{1}$ and $\mathrm{X}_{4}$ have a low level multicollinearity, the method of selecting independent variables by stepwise regression yields a value of the regression coefficient for every independent variable similar to the value specified in the model, and the highest percentage of models that are correctly selected. In the case of the Tabu Search using either of the two objective functions a value of the regression coefficient is obtained for the independent variables $\mathrm{X}_{1}, \mathrm{X}_{3}$ and $\mathrm{X}_{4}$ higher than that specified in the model and the percentage of models correctly selected is a little lower than with the method of stepwise regression. Apart from this, it is found that the percentage of models correctly selected increases when the sample size increases both with the Tabu Search and the method of stepwise regression.

In the case that the independent variables $X_{1}$ and $X_{4}$ have a medium or high level multicollinearity, the selection of independent variables by the method of Tabu Search, using either of the two objective functions yields a value of the regression coefficient for every independent variable similar to the value specified in the model in all circumstances, and the percentage of models correctly selected is higher than with the method of stepwise regression. Apart from this, it is found that the percentage of models correctly selected increases when the sample size increases and is similar to that when the value of the correlation coefficient among independent variables $X_{1}$ and $X_{4}$ changed. When the independent variables are selected by the method of stepwise regression, this gives a regression coefficient for the independent variables that indicated a difference from that specified in the model. Apart from this, it is found that the value of the regression coefficient for the independent variables $X_{1}$ and $X_{4}$ has a value higher than that specified in the model in all circumstances. In addition, it is found that the percentage of models correctly selected increases when the sample size increases, but the value of the correlation coefficient among independent variables $\mathrm{X}_{1}$ and $\mathrm{X}_{4}$ decreases. 


\section{References}

Bruce, L. B., David, A,.D., \& Richard, T. O. (1990). Linear Statistical models: an applied approach. (2 ${ }^{\text {nd }}$ ed.),

Boston, USA: Duxbury Press.

Drezner, Z. \& George, A. (1999). Tabu Search Model Selection in Multiple Regression Analysis. Communication in Statistics-Simulation and Computation, 28(2): 349 - 367.

Drezner, Z., Marcoulides, G.A. \& Stohs, M.H. (2001). Financial Applications of a Tabu Search Variable Selection Model. Journal of Applied Mathematics and Decision Sciences, 5(4): 215 - 234.

Efroymson, M.A. (1960). Multiple regression analysis: Mathematical methods for digital computers. New York, USA: Wiley.

Eksioglu, B., Demirer, R., \& Capar, I. (2005). Subset selection in multiple linear regression: a new mathematical programming approach. Computer \& Industrial Engineering, 49(1): 55-167.

Glover, F. (1990). Tabu search: A tutorial. Interface, 20(4): 74-94.

Gujarati, D.N. (2009). Basic Econometrics. New York, USA: McGraw-Hill.

Holland, J. (1975). Adaptation in Natural and Artificial Systems. MA, USA: The University of Michigan Press.

Knox, J. (1989). The application of tabu search to the symmetric traveling salesman problem. A dissertation for the Philosophy of Business. University of Colorado, USA.

Marcoulides, G.A. \& Drezner, Z. (2004). Tabu Search Variable Selection with Resource Constraints. Communication in Statistics: Simulation and Computation, 33(2): $355-362$.

Mills, J.D., Olejnik, S.F., \& Marcoulides, G.A. (2005). The Tabu Search Procedure: An Alternative to the Variable Selection Methods. Multivariate Behavioral Research, 40(3): 351 - 371.

Montgomery, D. C., Peck, E. A., \& Vining, G. G. (2006). Introduction to linear regression analysis. (4 ${ }^{\text {th }}$ ed.), New Jersey, USA: John Wiley \& Sons, Inc.

Pacheco, J., Casado, S., \& Nunez, L. (2009). A variable selection method based on Tabu Search for logistic regression models. European Journal of Operational Research (EJOR), 199(2): 506-511.

Piriyakul, M. (1986). Regression Analysis. Bangkok, Thailand: Chuanpim Press.

Sacchi, L. H., \& Armentano, V. A. (2011). A Computational study of parametric tabu search for $0-1$ mixed integer programs. Computer \& Operational Research, 38(12): 464-473.

Seenoi, P. (2010). Test Statistics for Selecting Multiple Linear Regression Models. A thesis for the degree of Master of Science in Statistics. National Institute of Development Administration, Thailand.

Shen, Q., Shi, W., \& Kong, W. (2010). Modified tabu search approach for variable selection in quantitative structure-activity relationship studies of toxicity of aromatic compound. Artificial Intelligence in Medicine, 49(2): 61-66.

Siary, P., \& Berthiau, G. (1997). Fitting of tabu search to optimize functions of continuous variables. International Journal for Numerical Methods in Engineering, 40(13): 2449-2457.

Smith, D., \& Richard, N. (1981). Applied regression analysis. ( $2^{\text {nd }}$ ed.), New York, USA: Wiley.

Turajlic, N., \& Dragovic, I. (2012). A hybrid metaheuristic based on variable neighborhood search and tabu search for the web service selection problem. Electronic Notes in Discrete Mathematics, 39(1): 145-152.

Wang, Y., Lu Z., \& Gloverl, F. (2013). Backbone guided tabu search for solving the UBQP problem. Journal of Heuristics, 19(4): $679-695$. 